\title{
COLLAPSE CAPACITY SPECTRA BASED ON AN IMPROVED INTENSITY MEASURE
}

\author{
S. Tsantaki ${ }^{1}$, and C. Adam ${ }^{1}$ \\ ${ }^{1}$ Unit of Applied Mechanics, University of Innsbruck \\ Technikerstr. 13, 6020 Innsbruck, Austria \\ \{styliani.tsantaki, christoph.adam\}@uibk.ac.at
}

Keywords: Averaged Spectral Acceleration, Collapse Capacity, Earthquake Excitation, Improved Intensity Measure, P-Delta Effect, Reduced Dispersion.

\begin{abstract}
A collapse capacity spectrum represents the seismic collapse capacity of highly inelastic non-degrading single-degree-of-freedom systems vulnerable to the destabilizing effect as a function of characteristic structural parameters. In this paper collapse capacity spectra with reduced dispersion due to record-to-record uncertainties are presented. This reduction is achieved utilizing an improved intensity measure, based on the spectral acceleration averaged in a certain period band between the structural period and an elongated period. It is shown that the "optimal" elongated period, which is related to the smallest achievable dispersion, depends primarily on the structural period. Based on extensive parametric studies, an analytical expression for the optimal ratio between elongated and structural period is derived. This analytical period ratio is constant for systems with a period larger than $0.15 \mathrm{~s}$. In several examples the superiority of the presented collapse capacity spectra with respect to the related dispersion is shown and quantified.
\end{abstract}




\section{INTRODUCTION}

The dispersion of the seismic response due to record-to-record (RTR) uncertainties depends on various factors such as the considered set of earthquake ground motions, the type of structure (regularity, etc.), the considered response quantity, and the choice of the intensity measure (IM).

In this paper the latter issue is addressed when predicting the collapse capacity of highly inelastic single-degree-of-freedom (SDOF) systems vulnerable to second-order effects of gravity. The considered systems exhibit a negative post-yield stiffness, however, material degradation is assumed to be negligible. Fundamental studies of the P-delta effect on inelastic SDOF systems subjected to strong motion earthquakes have been presented e.g. in $[1,2]$. Recently, in the research group of the authors of the subsequently presented study the collapse capacity of P-delta sensitive SDOF systems has been assessed qualitatively and quantitatively for a set of characteristic parameters [3,4]. The presentation of the collapse capacity and its dispersion has been referred to as collapse capacity spectra, providing a compact and easily applicable tool for the practicing engineer [3]. In [3,4] the 5\% damped spectral pseudoacceleration at the structural period serves as IM. In [5] the effect of other "standard" IMs on the collapse capacity has been investigated. However, the so far applied IMs result in a quite large dispersion of the collapse capacity.

In this study, the influence of a recently proposed IM [6] on the RTR randomness of collapse capacity spectra is investigated by conducting extensive parametric Incremental Dynamic Analyses (IDAs) [7]. Following an idea of [6] the geometric mean of pseudo-spectral acceleration ordinates over a certain range of periods is employed as IM, taking into consideration the period elongation of inelastic SDOF systems. A slightly modified version of this IM is applied, at which the structure's period serves as the lower limit of this period range. In [8] it has been shown that for the considered systems the "optimal" period range, i.e. the period range leading to the smallest dispersion, depends highly on the structure's period. Thus, the optimal period range is identified as a function of the structure's period. In [9] collapse fragility curves are presented, and compared with outcomes of the corresponding benchmark study [3] on the same systems, based on the 5\% damped spectral pseudo-acceleration at the structural period as IM. As a main outcome of the presented investigation an analytical formulation of the "optimal" elongated period is derived, which is a function of the structural period only. Simultaneously, the dependence of the RTR variability on the viscous damping coefficient is investigated.

\section{DEFINITIONS AND FRAMEWORK}

\subsection{P-delta effect on an inelastic SDOF System}

In an inelastic SDOF system the gravity load generates a shear deformation of its hysteretic force-displacement relationship. Characteristic displacements (such as the yield displacement) of this relationship remain unchanged, whereas the characteristic forces (such as the strength) are reduced. As a result, the slope of the curve is decreased in its elastic and post-elastic branch of deformation. The magnitude of this reduction can be expressed by means of the socalled stability coefficient $\theta$ [2]. $\theta$ is a function of the gravity load, geometry, and stiffness. Figure 1 visualizes the P-delta effect on the non-dimensional hysteretic behavior of a SDOF system with non-deteriorating bilinear characteristics. In this example, the post-yield stiffness is negative, because the stability coefficient $\theta$ is larger than the hardening ratio $\alpha$.

A negative slope of the post-yield stiffness, expressed by the difference of the stability coefficient $\theta$ and the strength hardening coefficient $\alpha, \theta-\alpha$, is the essential condition that the 
structure may collapse under severe earthquake excitation. In [3] it is shown that collapse of inelastic SDOF systems vulnerable to P-delta is mainly governed by the following parameters:

- The negative slope of the post-yield stiffness expressed by $\theta-\alpha$,

- the elastic structural period of vibration $T_{1}$,

- the viscous damping coefficient $\zeta$ (usually $5 \%$ ), and

- the shape of the hysteretic loop.

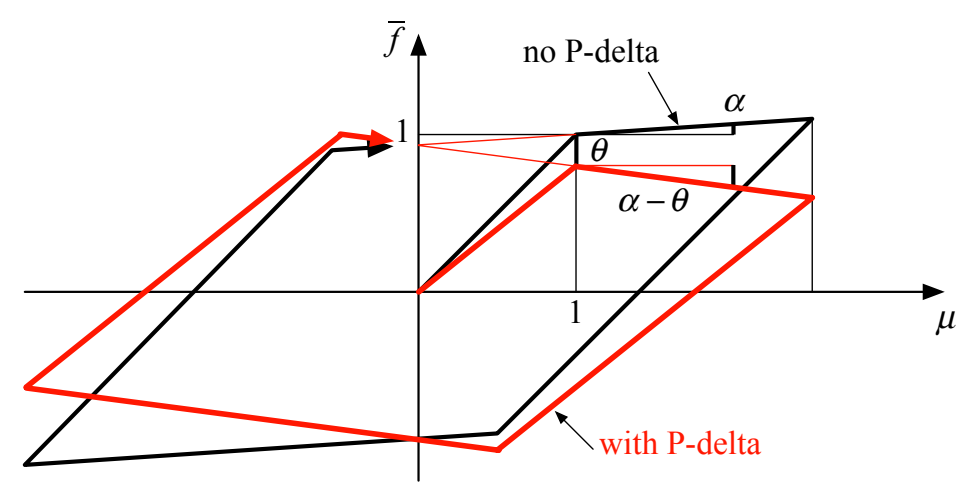

Figure 1: Normalized bilinear cyclic behavior with and without destabilizing effect of gravity loads [3].

\subsection{Utilized intensity measure}

In the present study, a slightly modified form of the IM defined in [6] is employed, which is based on the geometric mean of the $5 \%$ damped spectral pseudo-acceleration over the period range $\Delta T$,

$$
\Delta T=T_{n}-T_{1}, \quad T_{n}>T_{1}
$$

between the structural period $T_{1}$ and an elongated period $T_{n}$ of $T_{1}$ (note that $T_{n}$ is not the $n$th structural period). At equally spaced discrete periods $T_{i}$ within of $\Delta T$,

$$
T_{i}=T_{1}+(i-1) \delta T, \quad i=1, \ldots, n \quad, \quad \delta T=\frac{\Delta T}{n-1}=\frac{T_{n}-T_{1}}{n-1}
$$

the corresponding discrete spectral pseudo-accelerations $S_{a}\left(T_{i}\right)(i=1, \ldots, n)$ are combined according to [6]

$$
S_{a, g m}\left(T_{1}, T_{n}\right)=\left(\prod_{i=1}^{n} S_{a}\left(T_{i}\right)\right)^{1 / n}
$$

An appropriate IM should comply with the following properties [6]:

- Hazard compatibility, i.e. the IM quantifies appropriately the ground motion hazard at the site

- Efficiency, which is defined as the dispersion of the IM values associated with a given response quantity level

- Sufficiency, i.e. the IM is conditionally statistically independent of ground motion characteristics such as magnitude, distance, epsilon, etc.

- Scaling robustness 
In [6] it is shown that $S_{a, g m}\left(T_{1}, T_{n}\right)$ is statistically independent from ground motion characteristics and scaling factors, and thus, it complies with the IM properties sufficiency and scaling robustness.

In the following considerations the accelerated efficiency of the IM $S_{a, g m}\left(T_{1}, T_{n}\right)$ with respect to the reduction of collapse capacity dispersion of P-delta sensitive SDOF systems due to the RTR variability is investigated, compared to the efficiency of the conventional IM $S_{a}\left(T_{1}\right)$ (spectral pseudo-acceleration at the period of the structure).

\subsection{Ground motion scaling}

In this study, RTR uncertainties are captured employing the 44 far-field ground motions of the ATC63-FF record set described in FEMA P-695 [10]. The records of the ATC63-FF set originate from severe seismic events of magnitude between 6.5 and 7.6 and closest distance to the fault rupture larger than $10 \mathrm{~km}$. Thereby, only strike-slip and reverse sources are considered. The 44 records of this set were recorded on NEHRP site classes C (soft rock) and D (stiff soil). For further details see [10].

To obtain comparable results, the ground motion records must be scaled. When using Equation 3 as IM, scaling of the original $j$ th ground acceleration $\ddot{x}_{g, \text { unscaled }}^{(j)}$ must be performed over the period range $\Delta T$ according to

$$
\ddot{x}_{g, \text { scaled }}^{(j)}=\frac{S_{a, g m}^{r e f}\left(T_{1}, T_{n}\right)}{S_{a, g m}^{(j)}\left(T_{1}, T_{n}\right)} \ddot{x}_{g, \text { unscaled }}^{(j)}
$$

to account for the spectral shape within $\Delta T$. Thereby, $S_{a, g m}^{r e f}\left(T_{1}, T_{n}\right)$ is the target mean spectral acceleration, which is the same for all ground motions of the considered record set.

\subsection{Collapse capacity}

The collapse capacity is defined as the maximum ground motion intensity at which the structure still maintains dynamic stability [11]. Most generally, the IDA procedure is applied to predict the collapse capacity. In an IDA for a given structure and a given acceleration time history of an earthquake record dynamic time history analyses are performed repeatedly, at which in each subsequent run the intensity of the ground motion is incremented [7]. As an outcome a characteristic IM is plotted against the corresponding maximum characteristic structural response quantity for each analysis. The procedure is stopped, when the response grows to infinity, i.e. structural failure occurs. The corresponding IM is referred to as collapse capacity of the considered structure for this specific ground motion record (subsequently denoted by $j$ ). In this study, the relative collapse capacity related to the $j$ th ground motion record corresponds to the suggested IM $S_{a, g m}^{(j)}\left(T_{1}, T_{n}\right)$ at collapse divided by the gravity and base shear coefficient $\gamma$,

$$
\left.C C_{j}\right|_{S_{a, g m}}=\frac{\left.S_{a, g m}^{(j)}\left(T_{1}, T_{n}\right)\right|_{\text {collapse }}}{g \gamma}, \gamma=\frac{f_{y}}{m g}
$$

$f_{y}$ is the yield strength, $m$ the mass of the SDOF system, and $g$ denotes the gravity. Since the inherent RTR variability leads to different collapse capacities for different ground motion records, the collapse capacities are determined for all records of a ground motion set, and subsequently evaluated statistically. In $[12,13]$ good arguments are provided for representing a set of corresponding collapse capacities by a log-normal distribution. A log-normal distribu- 
tion is characterized by the median of the individual collapse capacities $C C_{i}$ (referred to as $C C$ ), and the 16th and 84th percentiles of the collapse capacities denoted as $C C_{16}$ and $C C_{84}$, respectively. In the following, the dispersion quantity $s^{*}[3]$

$$
s^{*}=\ln \sqrt{s_{u} s_{l}}, s_{u}=\frac{C C_{84}}{C C}, s_{l}=\frac{C C}{C C_{16}}
$$

is utilized as a representative measure of the RTR variability of the individual collapse capacities. Note that in the log-domain $s^{*}$ corresponds to the standard deviation, $\sigma \approx s^{*}$ [14].

\subsection{Collapse capacity spectrum}

In a collapse capacity spectrum the representative collapse capacity of a P-delta sensitive and non-deteriorating SDOF system with given negative post-yield stiffness ratio $\theta-\alpha$, viscous damping $\zeta$, and hysteretic loop is presented as a function of the structural period $T_{1}$ [3]. As an example, Figure 2 shows 5\% damped median collapse capacity spectra in the period range from $T_{1}=0.1$ to $T_{1}=5 \mathrm{~s}$ based on the conventional IM $S_{a}\left(T_{1}\right)$ for bilinear SDOF systems with various negative post-yield stiffness ratios $\theta-\alpha$. These spectra are based on the 44 records of the ATC63-FF ground motion set [3].

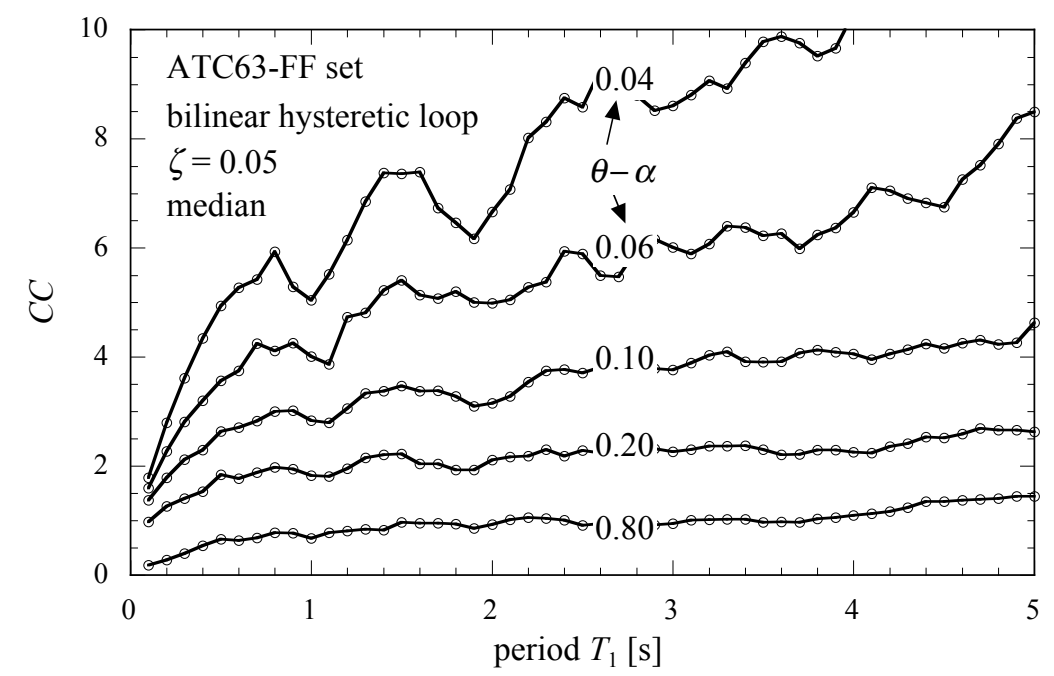

Figure 2: Median collapse capacity spectra $C C$ based on the conventional IM $S_{a}\left(T_{1}\right)$ [3].

\section{DISPERSION OF THE COLLAPSE CAPACITY}

\subsection{Record-to-record variability for various intensity measures}

Subsequently, the effect of the proposed IM $S_{a, g m}\left(T_{1}, T_{n}\right)$ on the dispersion parameter $s^{*}$ of the collapse capacity, Equation 5, is investigated. In particular it is of interest to reveal the impact of the period band $\Delta T$ on $s^{*}$ in dependence of the characteristic structural parameters specified in Section 2.1. As reference solution serve the outcomes based on the conventional IM, i.e. the 5\% damped spectral acceleration $S_{a}\left(T_{1}\right)$ at the initial structural period $T_{1}$ [3]. All subsequent studies are based on bilinear cyclic behavior of the SDOF system. Viscous damping $\zeta$ is set to $5 \%$, unless otherwise indicated.

In an initial study SDOF systems exhibiting a negative post-yield stiffness ratio of $\theta-\alpha=0.20$ and structural periods $T_{1}$ in the range between 0 and $5.0 \mathrm{~s}$ are considered. For each structural configuration $\left(T_{1}\right)$ the collapse capacity is determined for all records of the 
ATC63-FF ground motion set by IDA, and parameter $s^{*}$ is evaluated. This analysis is conducted for period bands $\Delta T=0.4 \mathrm{~s}, 0.6 \mathrm{~s}, 0.8 \mathrm{~s}, 1.0 \mathrm{~s}, 1.2 \mathrm{~s}$, and $1.4 \mathrm{~s}$.

A graphical representation of the derived dispersion parameter $s^{*}$ with respect to the structural period $T_{1}$ is shown in Figure 3 [9]. The solid line in red corresponds to $s^{*}$ of the original base case study [3] using the conventional IM $S_{a}\left(T_{1}\right)$. Black lines provided with different markers represent outcomes utilizing the proposed IM according to Equation 3 for various period ranges $\Delta T$, as specified in the figure. For very stiffness systems up to period of $T_{1}=0.22 \mathrm{~s}$ the conventional IM renders the smallest dispersion. As discussed in [4], a rigid system $\left(T_{1}=0\right)$ does not exhibit any aleatory uncertainty, i.e. $s^{*}=0$, when using the spectral acceleration at $T_{1}$ as IM. However, this IM leads to the largest dispersion for moderately stiff and flexible systems with $T_{1}>0.8 \mathrm{~s}$. If the structural period $T_{1}$ becomes larger, the accelerated efficiency of the proposed IM in reducing the dispersion $s$ becomes obvious. While for systems with initial periods between 0.22 and $0.9 \mathrm{~s}$ the period band $\Delta T=0.4 \mathrm{~s}$ for averaging the spectral acceleration leads to the smallest dispersion, for more flexible systems with $T_{1}>1.58 \mathrm{~s}$ the period band $\Delta T=1.4 \mathrm{~s}$ gives the smallest dispersion. However, the difference between the outcomes based on $\Delta T=1.2 \mathrm{~s}$ and $\Delta T=1.4 \mathrm{~s}$ is negligible. It is interesting to note that for this particular structural configuration efficiency of the proposed IM is largest for systems with periods $T_{1}$ between $2.6 \mathrm{~s}$ and $3.0 \mathrm{~s}$. Here, the reduction of $s^{*}$ is more than $50 \%$, compared to the outcome using the conventional IM. For larger periods the ability of IM $S_{a, g m}\left(T_{1}, T_{n}\right)$ to decrease $s^{*}$ becomes lower, because acceleration response spectra become almost horizontal for such flexible systems.

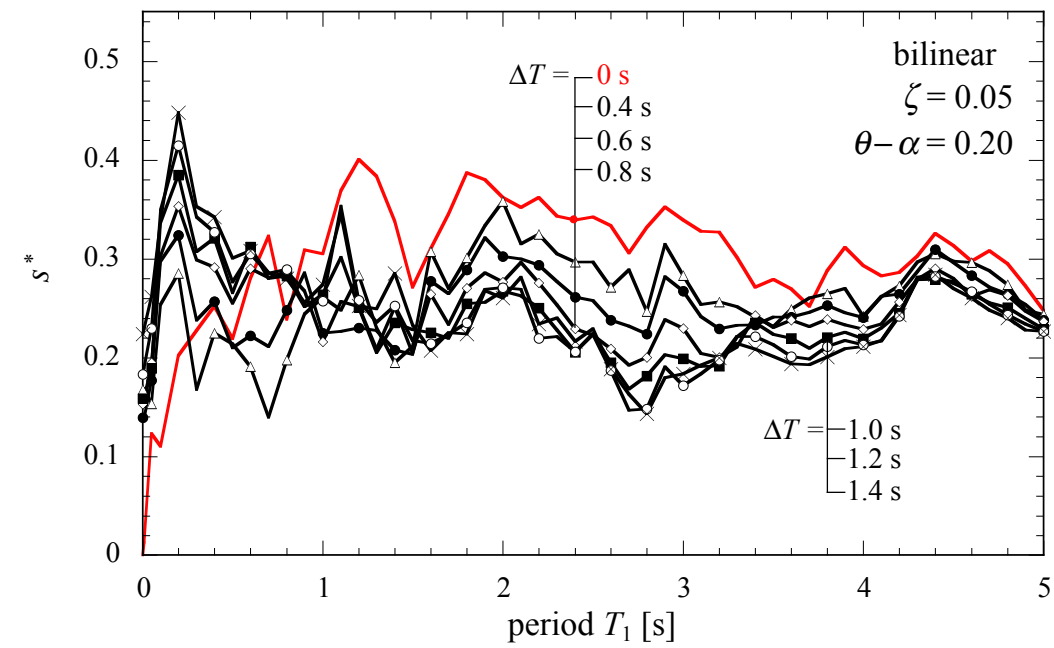

Figure 3: Dispersion parameter $s^{*}$ of the collapse capacity as a function of the structural period $T_{1}$ for different period ranges $\Delta T$. Discrete negative post-yield stiffness ratio $\theta-\alpha=0.20$.

In order to reveal a general trend of the effect of the proposed IM on the dispersion parameter $s^{*}$ in a further step the study is extended considering a series of negative post-yield stiffness ratios $\theta-\alpha$. In particular, the following discrete values of $\theta-\alpha$ are assigned to the SDOF systems: $\theta-\alpha=0.04,0.06,0.08,0.10,0.20,0.30,0.40,0.50,0.60,0.80$. Additionally, period bands $\Delta T$ of $1.8 \mathrm{~s}, 2.4 \mathrm{~s}, 3.0 \mathrm{~s}$, and $4.0 \mathrm{~s}$ are taken into account. Then, from the individual outcomes of $s^{*}$ mean values with respect to the negative post-yield stiffness ratio $\theta-\alpha$ and to the period $T_{1}$, respectively, are determined.

Figure 4 shows the mean of $s^{*}$, derived from all individual outcomes comprising the entire set of parameter $\theta-\alpha$, for each IM separately as a function of the structural period. The solid 
line in red corresponds to the mean of $s^{*}$ based on the conventional IM $S_{a}\left(T_{1}\right)$, whereas lines in black and blue represent the mean dispersion parameter for different period bands $\Delta T$ of the proposed IM. The results of this figure confirm in a large extent the findings of Figure 3. Except for very stiffness structures, the conventional IM leads to a mean dispersion $s$ of the collapse capacity of about 0.4 , while the dispersion $s^{*}$ with the underlying proposed IM exhibits an average minimum of about 0.25 , considering only the most efficient period band $\Delta T$ at different periods $T_{1}$. From the results of this figure it can be concluded that there is no "optimal" IM for the entire range of structural periods, because the "optimal" period band $\Delta T$ is a function of $T_{1}$. I.e., the larger $T_{1}$ becomes, the larger is the required $\Delta T$ to render the minimum achievable dispersion parameter $s^{*}$. The relation between $T_{1}$ and the "optimal" period band $\Delta T$ will later be studied in more detail.

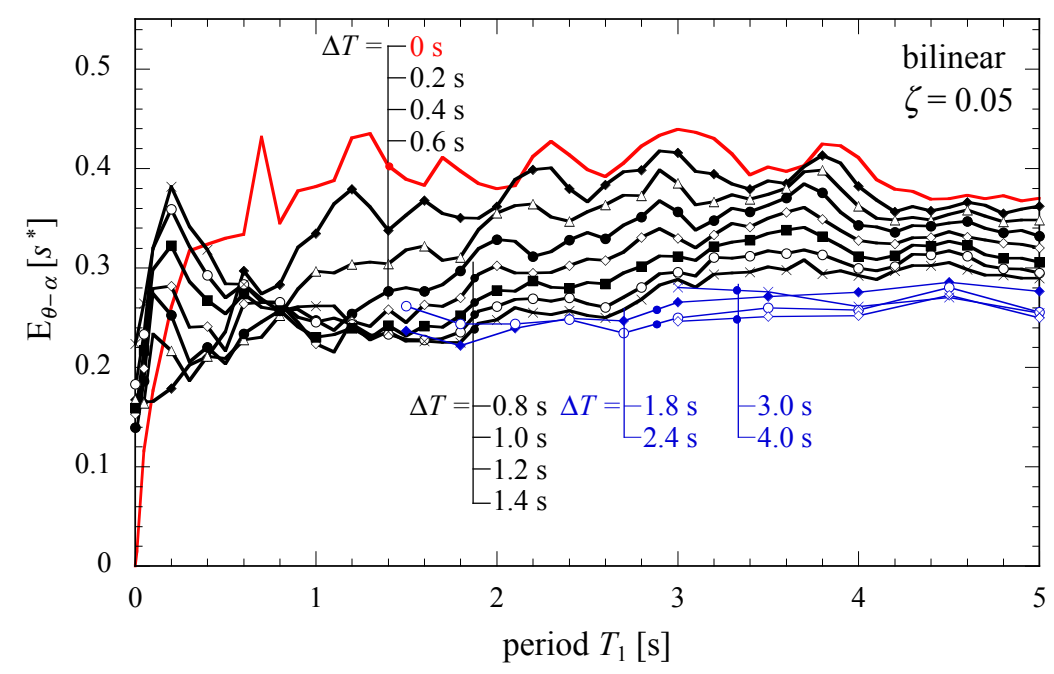

Figure 4: Mean values of the dispersion parameter $s^{*}$ with respect to the considered discrete post-yield stiffness ratios $\theta-\alpha$ as a function of the structural period $T_{1}$ for various period ranges $\Delta T$.

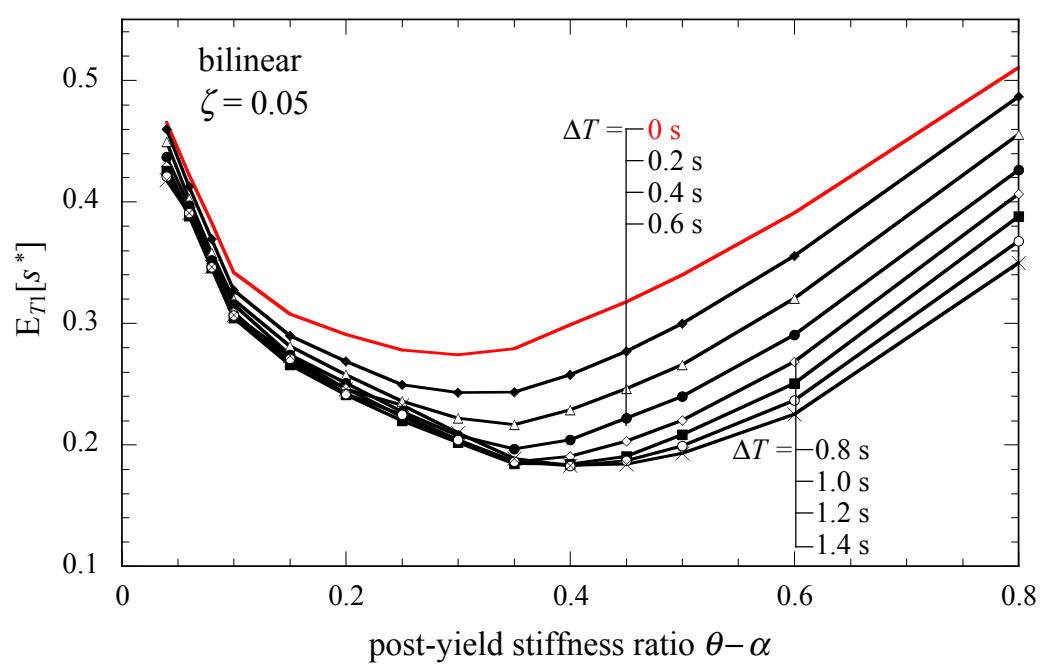

Figure 5: Mean values of the dispersion parameter $s^{*}$ with respect to the structural period range $0 \leq T_{1} \leq 5 \mathrm{~s}$ as a function of the post-yield stiffness ratio $\theta-\alpha$ for various period ranges $\Delta T$. 

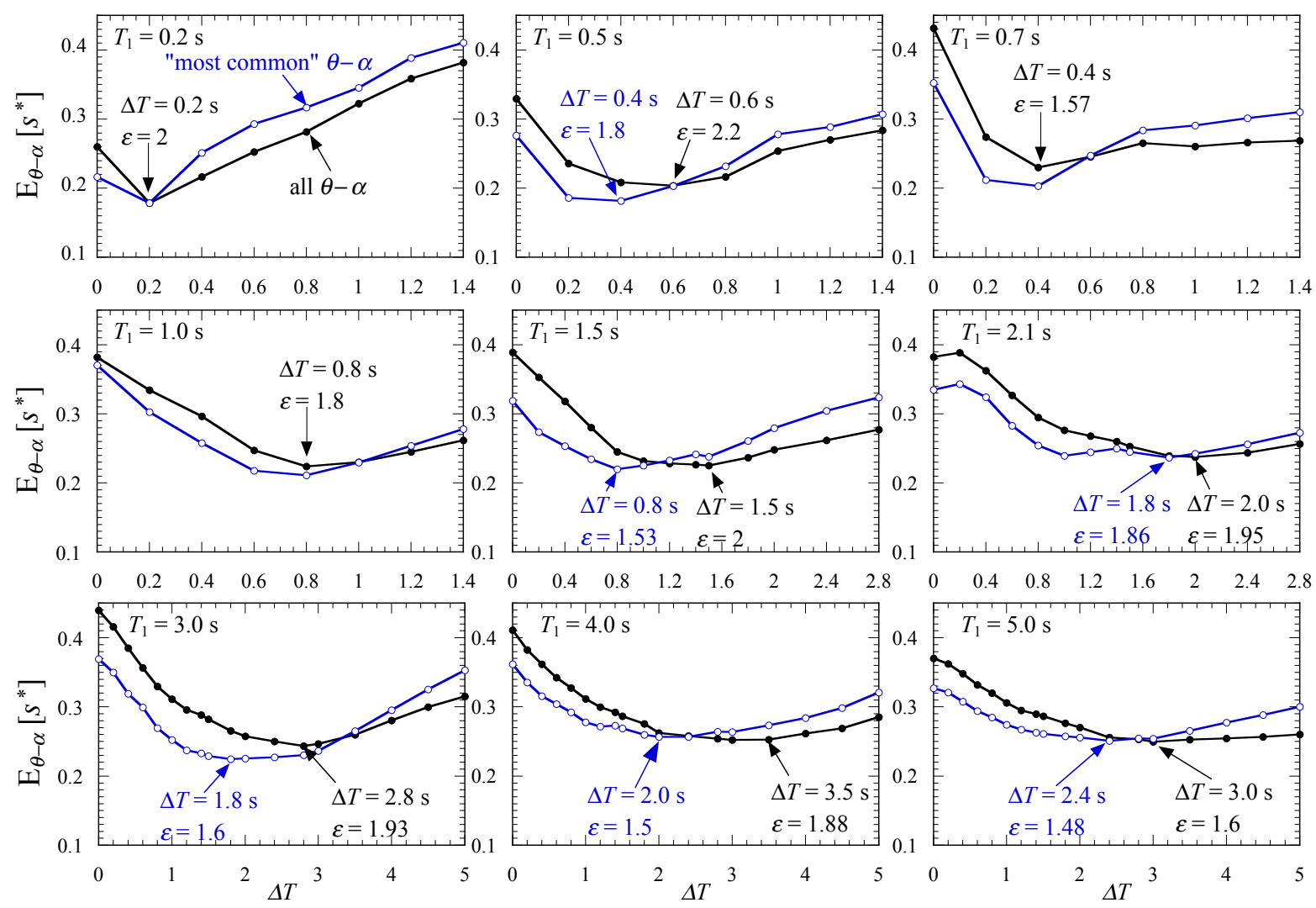

Figure 6: Mean values of the dispersion parameter $s^{*}$ with respect to the post-yield stiffness ratios $\theta-\alpha$ as a function of period range $\Delta T$ for nine discrete structural periods $T_{1}$. Black graphs: mean from all considered discrete values of $\theta-\alpha$. Blue graphs: mean from discrete values of $\theta-\alpha$ in the range of $0.08 \leq \theta-\alpha \leq 0.40$.

However next, the overall trend of the dispersion parameter $s^{*}$ with respect to the negative post-yield stiffness ratio $\theta-\alpha$ is discussed for IMs with different $\Delta T(0 \leq \Delta T \leq 1.4 \mathrm{~s})$. Figure 5 displays mean values of $s^{*}$ with respect to the structural period $T_{1}$ (in the range between 0 and $5.0 \mathrm{~s}$ equally spaced with increments of $0.1 \mathrm{~s}$ ) as a function of the negative post-yield stiffness ratio $\theta-\alpha$. In this representation, the conventional IM leads to the poorest performance in the entire range of $\theta-\alpha$. Thereby, the minimum mean dispersion $s^{*}$ of 0.275 exhibits the systems with a negative post-yield stiffness ratio of 0.30 . With increasing period band $\Delta T$ the achievable minimum of mean $s^{*}$ is reduced up to 0.18 , and the minimum is shift to $\theta-\alpha=0.40$ (for $\Delta T=1.4 \mathrm{~s}$ ). It is readily observed that for small $\theta-\alpha(\theta-\alpha<0.10)$ the reduction of mean $s^{*}$ (based on IM $S_{a, g m}\left(T_{1}, T_{n}\right)$ ) is quite small compared to the corresponding outcomes for the conventional IM $S_{a}\left(T_{1}\right)$.

Figure 6 shows for nine discrete structural periods $\left(T_{1}=0.2 \mathrm{~s}, 0.5 \mathrm{~s}, 0.7 \mathrm{~s}, 1.0 \mathrm{~s}, 1.5 \mathrm{~s}, 2.1 \mathrm{~s}\right.$, $3.0 \mathrm{~s}, 4.0 \mathrm{~s}, 5.0 \mathrm{~s}$, respectively) the mean dispersion parameter $s^{*}$ (with respect to $\theta-\alpha$ ) plotted against period band $\Delta T$ of the underlying proposed IM $S_{a, g m}\left(T_{1}, T_{n}\right)$. Thereby, on the one hand the mean of $s^{*}$ is determined comprising the complete set of considered post-yield stiffness ratios $\theta-\alpha$. The corresponding results are depicted in black. On the other hand, only the collapse capacities of $\theta-\alpha$ in the range of $0.08 \leq \theta-\alpha \leq 0.40$ (referred to as "most common $\theta-\alpha)$ are used to calculate the mean dispersion parameter $s^{*}$. Blue graphs represent these outcomes. In this set of $\theta-\alpha$ extreme values are excluded, which might distort the general trend of the response behavior. Exemplarily, Figure 7 shows for a system with $T_{1}=1.5 \mathrm{~s}$ parameter $s^{*}$ both for discrete $\theta-\alpha$ values and the proposed mean values. It can be readily observed that the trend of the dispersion for systems with an extremely large ratio of 
$\theta-\alpha=0.80$ is very different from the other depicted cases. The results of Figure 6 confirm that the "optimal" period band $\Delta T$ strongly depends on the structural period $T_{1}$. As already observed in Figure 4, the optimal value of $\Delta T$ becomes larger with increasing $T_{1}$. In all subplots period band $\Delta T$ leading to the minimum mean dispersion is specified. For example, for $T_{1}=1.0 \mathrm{~s}$ the minimum mean dispersion is 0.21 (all $\theta-\alpha$ ), compared to 0.38 for the conventional IM (i.e. $\Delta T=0$ ). For the minimum mean $s^{*}$ the corresponding $\Delta T$ is about $0.8 \mathrm{~s}$. It is interesting to note that the ratio $\varepsilon$ between the elongated period $T_{n}$ and initial structural $T_{1}$,

$$
\varepsilon=\frac{T_{n}}{T_{1}}
$$

lies for "optimal" $\Delta T$ in the range between 1.4 and 2.0.

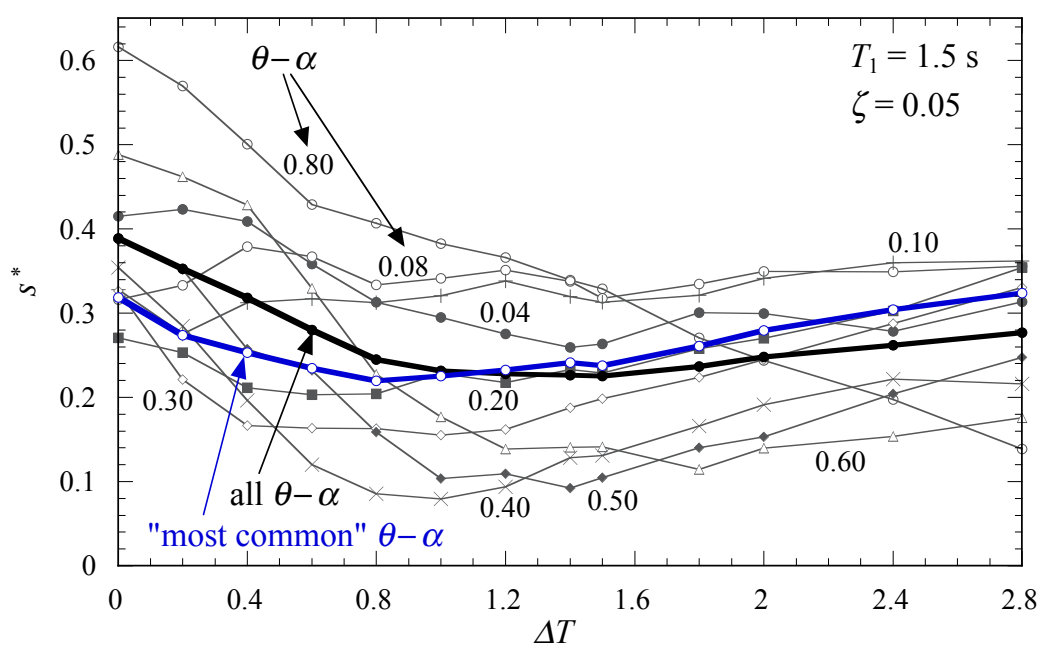

Figure 7: Dispersion parameter $s^{*}$ for various post-yield stiffness ratios $\theta-\alpha$ as a function of period range $\Delta T$. Structural periods $T_{1}=1.5 \mathrm{~s}$. Black graph: mean from all considered discrete values of $\theta-\alpha$. Blue graph: mean from discrete values of $\theta-\alpha$ in the range of $0.08 \leq \theta-\alpha \leq 0.40$.

\section{2 “Optimal" intensity measure}

Based on the observation of the results presented in Figure 6, in the subsequent section an attempt is made to derive a simple analytical expression for an "optimal" IM $S_{a, g m}\left(T_{1}, T_{n}\right)$, which leads globally to the smallest dispersion of the collapse capacities. Starting point is the consideration the "optimal" (i.e. the minimum) dispersion parameter $s^{*}$ for IM $S_{a, g m}\left(T_{1}, T_{n}\right)$, identified from extensive parametric IDA studies. In Figure 8 the mean of this quantity with respect to the considered negative post-yield stiffness parameters $\theta-\alpha$ is shown in solid lines as a function of the structural period $T_{1}$. Additionally to $5 \%$ damped SDOF systems also the outcomes for undamped and $2 \%$ damped systems are displayed. It is readily observed that the minimum of mean $s^{*}$ is in average about 0.24 in almost the entire period range $T_{1}$. This value remains the same considering the complete set of $\theta-\alpha$ and, alternatively, the set including the "most common" $\theta-\alpha$. Additionally, dashed lines represent the mean dispersion of the collapse capacities based on the conventional IM $S_{a}\left(T_{1}\right)$. Here, the mean $s^{*}$ is in average about 0.4 , which proofs the superiority of the proposed IM $S_{a, g m}\left(T_{1}, T_{n}\right)$ in reducing the RTR uncertainty. Furthermore, the results of Figure 8 show that the dispersion is globally unaffected from the amount of viscous damping, independent from the underlying IM. 


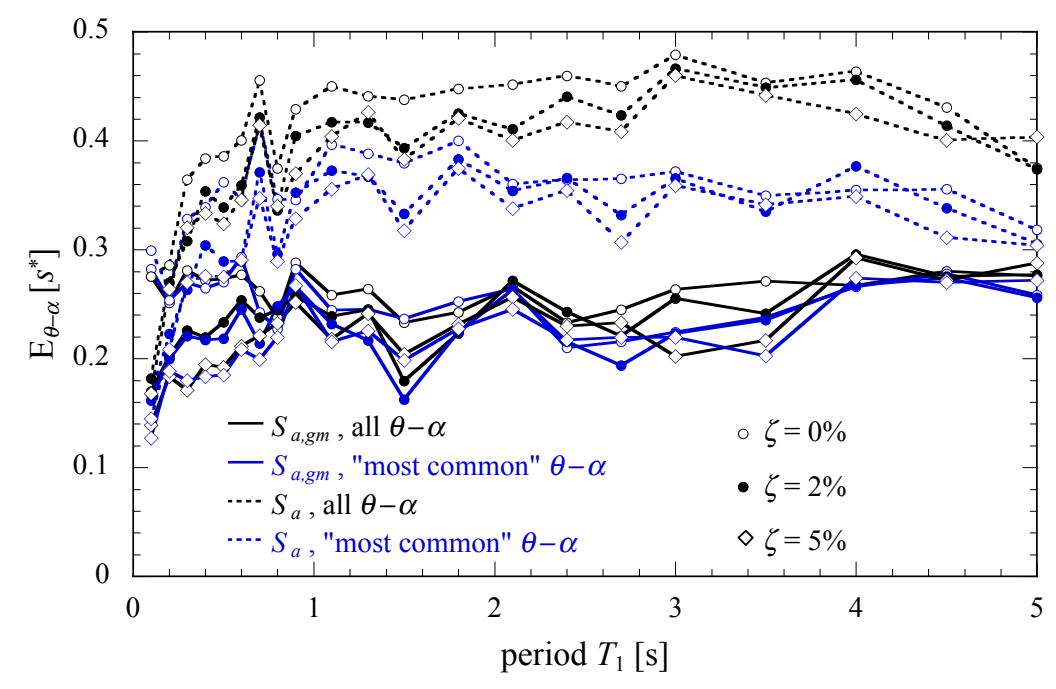

Figure 8: Mean with respect to the considered set of negative post-yield stiffness ratios $\theta-\alpha$ of the dispersion parameter $s^{*}$ as a function of $T_{1}$. Dispersion based on the conventional IM $S_{a}\left(T_{1}\right)$, and smallest obtained dispersion parameter based on the proposed IM $S_{a, g m}\left(T_{1}, T_{n}\right)$. Results for viscous damping of $0 \%, 2 \%$, and $5 \%$.

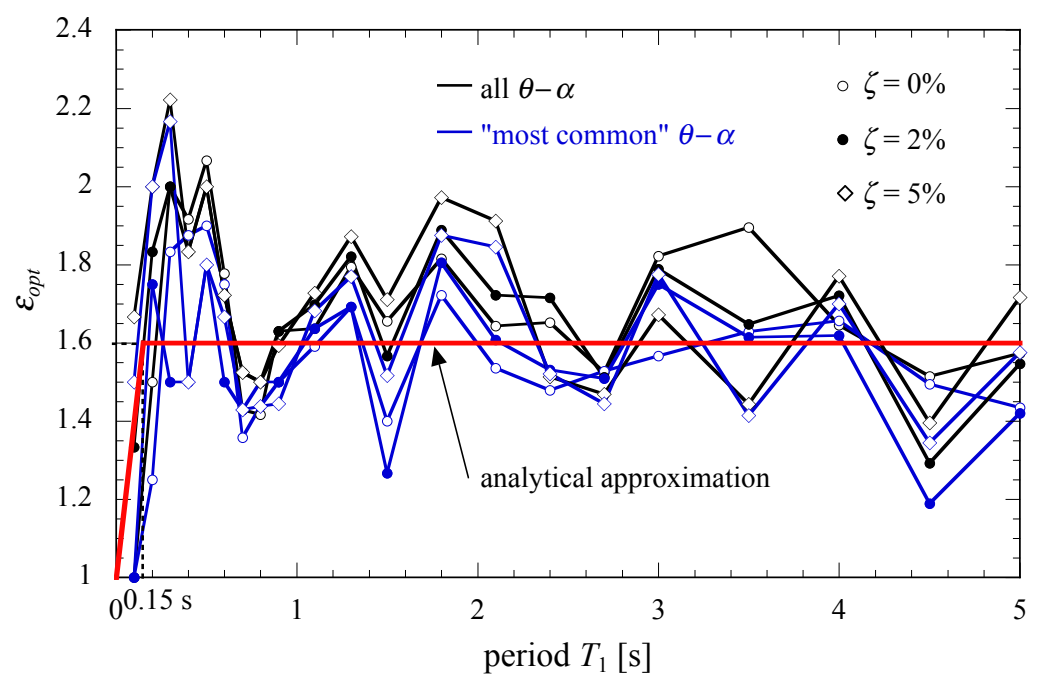

Figure 9: Ratio $\varepsilon_{\text {opt }}$ between the "optimal" elongated period $T_{n}$ and the initial structural period $T_{1}$ leading to the minimum mean dispersion $s$ as a function of $T_{1}$. Results for viscous damping of $0 \%, 2 \%$, and $5 \%$.

Figure 9 shows the ratios (according Equation 7) between the "optimal" elongated period $T_{n, o p t}$ and the initial period $T_{1}$,

$$
\varepsilon_{\text {opt }}=\frac{T_{n, o p t}}{T_{1}}
$$

as a function of $T_{1}$. Thereby, the "optimal" elongated period $T_{n, o p t}$ corresponds to the minimum of the mean dispersion $s^{*}$ displayed in Figure 8. It is readily observed that for rigid systems this ratio is 1 , because for this structure type the dispersion is 0 when using the conventional IM $S_{a}\left(T_{1}\right)$, as already discussed before [4]. Then follows a sharp rise of $\varepsilon_{\text {opt }}$ up to 2.2. For periods $T_{1}>0.8 \mathrm{~s} \varepsilon_{\text {opt }}$ fluctuates around a mean value of 1.6.

Based on this observation, the following analytical approximation for $\varepsilon_{\text {opt }}$, 


$$
\varepsilon_{a}\left(T_{1}\right)=\left\{\begin{array}{cl}
4 T_{1}+1 & 0 \leq T_{1} \leq 0.15 \mathrm{~s} \\
1.6 & T \geq 0.15 \mathrm{~s}
\end{array}\right.
$$

is proposed in an effort to provide for a simple but reliable "optimal" IM $S_{a, g m}\left(T_{1}, T_{n}\right)$. This simple expression is independent of viscous damping parameter $0 \leq \zeta \leq 0.05$, and negative post-yield stiffness ratio $\theta-\alpha$. Thus, the recommended elongated period for the proposed IM is determined according to

$$
T_{n, a}=\varepsilon_{a} T_{1}
$$

Consequently, the corresponding $\operatorname{IM} S_{a, g m}\left(T_{1}, T_{n, a}\right)$ is subsequently referred to "recommended IM".

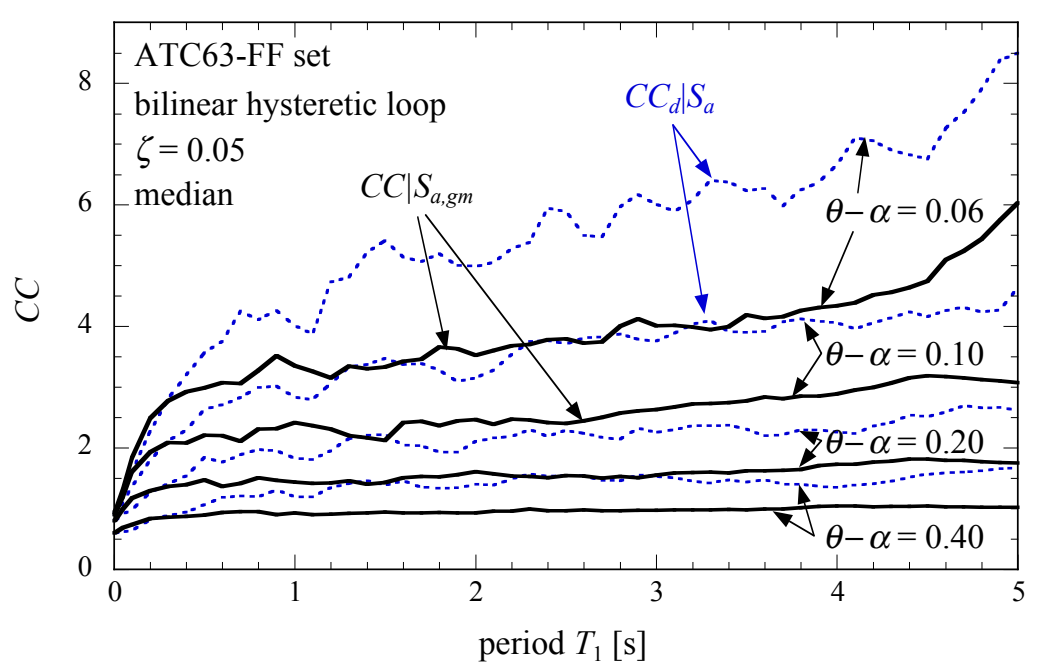

Figure 10: Median collapse capacity spectra for four different negative post-yield stiffness ratios $\theta-\alpha$. Solid black lines: spectra based on the recommended IM $S_{a, g m}\left(T_{1}, T_{n, a}\right)$. Dashed blue lines: spectra based on the conventional IM $S_{a}\left(T_{1}\right)$.

\section{COLLAPSE CAPACITY SPECTRA BASED ON "OPTIMAL" INTENSITY MEASURE}

Figure 10 shows median collapse capacity spectra for four selected post-yield stiffness ratios $\theta-\alpha=0.06,0.10,0.20$, and 0.40 . Viscous damping $\zeta$ is set to $5 \%$. Spectra displayed by solid black lines are based on the recommended IM $S_{a, g m}\left(T_{1}, T_{n, a}\right)$, compare also with Equation 10. Additionally, the corresponding "conventional" collapse capacity spectra, relying on the spectral acceleration $S_{a}\left(T_{1}\right)$ at the initial period as IM, are set in contrast. Dashed blue lines represent these spectra. At $T_{1}=0$ enhanced and conventional spectra coincide, since for rigid structures the period ratio $\varepsilon_{a}=1$, see Equation 9. Enhanced median spectra exhibit in the period range $T_{1}>0.2 \mathrm{~s}$ a smaller median collapse capacity compared to the conventional counterparts. This is a result of "averaging" the spectral acceleration in the period band $\Delta T$ in the descending branch of this quantity. However, the trend of the enhanced spectra with respect to $T_{1}$ remains similar to the original ones.

In Figure 11 dispersion parameters $s^{*}$, which correspond to these median spectra, are plotted against the initial structural period $T_{1}$. It is readily observed that for systems with $T_{1}=0$ the dispersion is zero, as discussed in [4]. However, stiff and more flexible systems exhibit for all considered structural configurations a reduced dispersion in the almost entire period range 
when using the proposed IM. This reduction becomes more pronounced the larger the negative post-yield stiffness ratio $\theta-\alpha$ is. While for $\theta-\alpha=0.06$ the decrease of $s^{*}$ is in average $15 \%$, for $\theta-\alpha=0.40$ parameter $s^{*}$ for IM $S_{a, g m}\left(T_{1}, T_{n, a}\right)$ is in general less than $50 \%$ of its counterpart for IM $S_{a}\left(T_{1}\right)$. This result shows that the dispersion magnitude not only depends on period $T_{1}$ but also on the negative post-yield stiffness ratio $\theta-\alpha$.
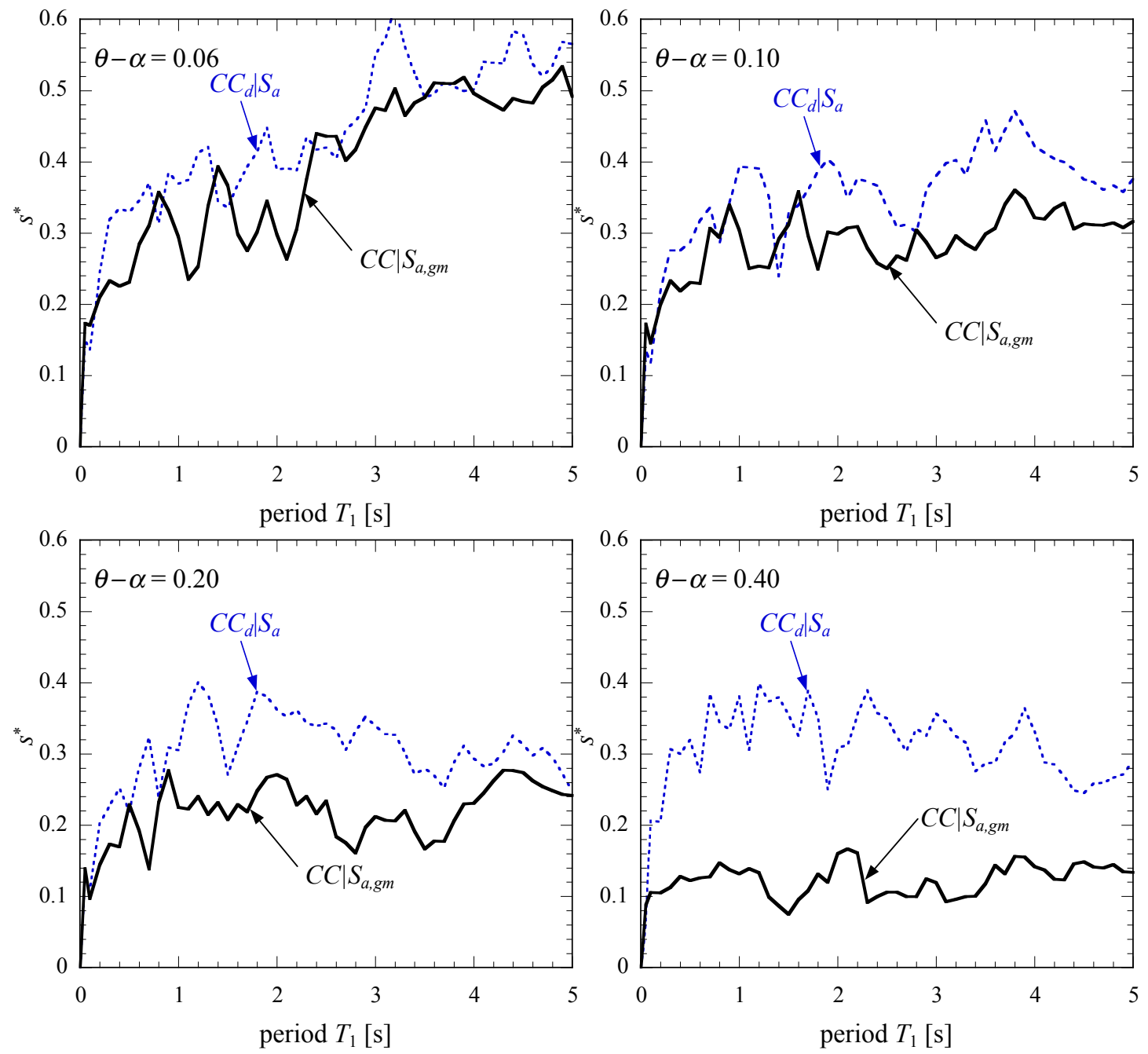

Figure 11: Dispersion parameter of the collapse capacities based on the recommended IM $S_{a, g m}\left(T_{1}, T_{n, a}\right)$ (solid black lines) and on the conventional IM $S_{a}\left(T_{1}\right)$ for four different negative post-yield stiffness ratios $\theta-\alpha$.

\section{CONCLUSIONS}

It has been shown that an intensity measure (IM) based on the averaged spectral acceleration of a certain period band between the structural period and a corresponding elongated period reduces considerably the record-to-record (RTR) variability of the seismic collapse capacity of highly inelastic single-degree-of-freedom systems vulnerable to the P-delta effect. From the results of extensive parametric studies the "optimal" elongated period has been identified. The "optimal" elongated period is related to the smallest achievable dispersion of a system with certain assigned characteristic structural parameters. It has been shown that this "optimal" period depends primarily on the initial structural period, and is more or less unaffected by viscous damping, and the negative post-yield stiffness ratio of the SDOF system. In general, the more flexible a system becomes, the larger is the period band between the elon- 
gated and the initial period that renders the smallest dispersion. However, this may not be the case for systems with a period larger than $3 \mathrm{~s}$, since in this period range the acceleration response spectrum is almost horizontal. An analytical expression of the ratio between the "optimal" elongated period and the structural period has been derived. As an outcome, this ratio is constant for systems with a period larger than $0.15 \mathrm{~s}$. In several applications, the reduction of the dispersion has been quantified. The largest reduction of the dispersion compared with the outcomes based on the conventional IM, i.e. the spectral acceleration at the initial structural period, can be achieved for systems with a negative post-yield stiffness ratio of 0.40 . For those systems, the dispersion is reduced by more than $50 \%$. In contrast, for systems with a mild negative post-yield stiffness ratio the reduction of the dispersion is quite small. The derived collapse capacity spectra based on the enhanced IM are valid for non-deteriorating bilinear cyclic behavior only. The effect of other constitutive laws such as pinching and peakoriented hysteretic loops on the RTR variability of the collapse capacity spectra will be addressed in further studies.

\section{ACKNOWLEDGEMENT}

S. Tsantaki conducted the research for this paper as a student of the Doctoral School (FWF DK-plus) "Computational Interdisciplinary Modelling", which is based at the University of Innsbruck and substantially funded by the Austrian Science Fund (FWF).

\section{REFERENCES}

[1] D. Bernal, Amplification factors for inelastic dynamic P- $\Delta$ effects in earthquake analysis. Earthquake Engineering and Structural Dynamics, 15, 635-651, 1987.

[2] G.A. MacRae, P- $\Delta$ effects on single-degree-of-freedom structures in earthquakes. Earthquake Spectra, 10, 539-568, 1994.

[3] C. Adam, C. Jäger, Seismic collapse capacity of basic inelastic structures vulnerable to the P-delta effect. Earthquake Engineering and Structural Dynamics, 41, 775-793, 2012.

[4] C. Jäger, C. Adam, Influence coefficients for collapse capacity spectra. Journal of Earthquake Engineering, accepted for publication.

[5] C. Adam, C. Jäger, Dynamic instabilities of simple inelastic structures subjected to earthquake excitation. H. Irschik, M. Krommer, A. Belyaev eds. Advanced Dynamics and Model-Based Control of Structures and Machines, pp. 11-18. Springer Wien, New York, 2012.

[6] M. Bianchini, P. Diotallevi, J.W. Baker, Prediction of inelastic structural response using an average of spectral accelerations. 10th International Conference on Structural Safety and Reliability (ICOSSAR 09), Osaka, Japan, September 13-19, 2009, 8 pp.

[7] D. Vamvatsikos, C.A. Cornell, Incremental dynamic analysis. Earthquake Engineering and Structural Dynamics, 31, 491-514, 2002.

[8] S. Tsantaki, C. Jäger, C. Adam, Improved seismic collapse prediction of inelastic simple systems vulnerable to the P-delta effect based on average spectral acceleration. $15^{\text {th }}$ World Conference on Earthquake Engineering (15 WCEE), Lisbon, Portugal, September 24-28, 2012, digital paper, paper no. 0287, 10 pp., 2012. 
[9] C. Adam, S. Tsantaki, Seismic collapse fragility of structures vulnerable to the P-delta effect. Submitted for publication $11^{\text {th }}$ International Conference on Structural Safety \& Reliability (ICOSSAR 2013), Columbia University, New York, June 16-20, 2013.

[10] FEMA P-695, Quantification of building seismic performance factors. Federal Emergency Management Agency, 2009.

[11] H. Krawinkler, F. Zareian, D.G. Lignos, L.F. Ibarra, Prediction of collapse of structures under earthquake excitations. M. Papadrakakis, N.D. Lagaros, M. Fragiadakis eds. 2nd International Conference on Computational Methods in Structural Dynamics and Earthquake Engineering (COMPDYN 2009), Rhodes, Greece, June 22-24, 2009, CDROM paper, paper no. CD449.

[12] L.F. Ibarra, H. Krawinkler, Global collapse of frame structures under seismic excitations. PEER Report 2005/06. University of California at Berkeley, California, Pacific Earthquake Engineering Research Center.

[13] L. Ibarra, H. Krawinkler, Variance of collapse capacity of SDOF systems under earthquake excitations. Earthquake Engineering and Structural Dynamics, 40, 12991314, 2011.

[14] E. Limpert, W.A. Stahel, M. Abbt, Log-normal distributions across the sciences: Keys and clues. BioScience, 51, 341-352, 2001. 